\title{
Development of digital organ-on-a-chip to assess hepatotoxicity and extracellular vesicle-based anti-liver cancer immunotherapy
}

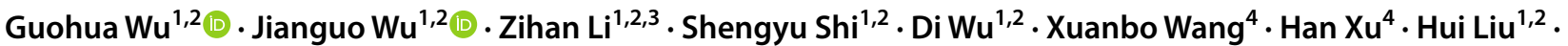 \\ Yixiao Huang $^{5,6} \cdot$ Rending Wang $^{1,7} \cdot$ Jia Shen ${ }^{1,7}$ (1) Z Zhihong Dong ${ }^{8} \cdot$ Shuqi Wang ${ }^{2,5,6,9}$
}

Received: 4 October 2021 / Accepted: 23 January 2022 / Published online: 3 March 2022

(c) The Author(s) 2022

\begin{abstract}
Organ-on-a-chip systems have been increasingly recognized as attractive platforms to assess toxicity and to develop new therapeutic agents. However, current organ-on-a-chip platforms are limited by a "single pot" design, which inevitably requires holistic analysis and limits parallel processing. Here, we developed a digital organ-on-a-chip by combining a microwell array with cellular microspheres, which significantly increased the parallelism over traditional organ-on-a-chip for drug development. Up to 127 uniform liver cancer microspheres in this digital organ-on-a-chip format served as individual analytical units, allowing for analysis with high consistency and quick response. Our platform displayed evident anti-cancer efficacy at a concentration of $10 \mu \mathrm{M}$ for sorafenib, and had greater alignment than the "single pot" organ-on-a-chip with a previous in vivo study. In addition, this digital organ-on-a-chip demonstrated the treatment efficacy of natural killer cell-derived extracellular vesicles for liver cancer at $50 \mu \mathrm{g} / \mathrm{mL}$. The successful development of this digital organ-on-a-chip platform provides high-parallelism and a low-variability analytical tool for toxicity assessment and the exploration of new anticancer modalities, thereby accelerating the joint endeavor to combat cancer.
\end{abstract}

\section{Graphic abstract}

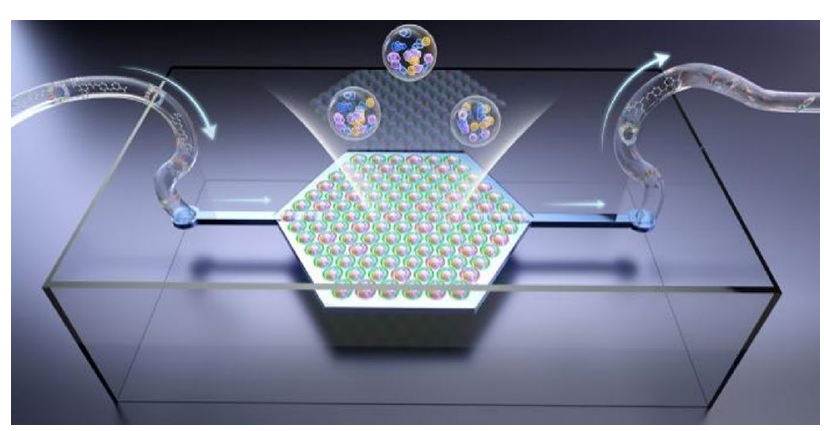

Keywords Digital · Organ-on-a-chip · Microwell array $\cdot$ Microspheres $\cdot$ Extracellular vesicles

\section{Introduction}

Jia Shen

jiashen@zju.edu.cn

$\triangle$ Zhihong Dong

zhdong@cdu.edu.cn

Shuqi Wang

shuqi@scu.edu.cn

Extended author information available on the last page of the article
Liver cancer, including primary and secondary malignancies, is one of the most common and lethal malignancies with high morbidity and mortality worldwide. Each year, the global incidence of liver cancer increases by more than 800,000 , and approximately 780,000 people die of this type of cancer [1]. Currently, only a few specific drugs are available to treat liver cancer [2], which highlights the urgent need 
to develop new drugs or other novel strategies, including biologics, functional materials, and extracellular vesicles for improved therapeutic outcomes. Although two-dimensional (2D) monolayer cultures and animal models are widely used for anticancer drug screening, the inability of these preclinical models to predict drug responses in human tumors in vivo has resulted in few preclinical successes translating into clinical practice $[3,4]$. Compared to in vivo conditions in humans, traditional 2D cultures lack the interactions between tumor cells, the extracellular matrix, surrounding vasculature, and the complex three-dimensional (3D) microenvironment, which are key for drug screening [5-8]. Although animal models enable drug screening, inherent intraspecific and physiological differences between humans and animals often undermine the success rates of drug development $[9,10]$. Hence, a drug screening platform that can better represent the tumor microenvironment is highly sought after to accurately predict toxicity and efficacy, and thus to achieve a higher success rate prior to the translation to clinical trials.

Organ-on-a-chip has recently become a powerful platform for drug and toxicological screening in vitro [11-16]. For example, Chen et al. developed a visual myocardial chip for drug development by assembling cardiomyocytes on morpho butterfly wings and filling the nanogaps of the wings with carbon nanotubes to increase the color contrast [17]. Nanotechnology such as anisotropic structural color particles (SCPs) [18] and 3D bioprinting [19] have further advanced organ-on-a-chip for drug testing in vitro. However, traditional organ-on-a-chip is based on the "single pot" method [20], in which drug test is a holistic analysis and the parallelism of testing is poor. Ma et al. placed hepatocytes (HepG2) and human aortic endothelial cells (HAEC) in different locations to form a bionic liver lobule on a chip to evaluate the liver toxicity of drugs [21]. In another study, Lu et al. used decellularized liver scaffolds and photocrosslinkable gelatin to mimic the extracellular matrix and constructed liver-on-a-chip to assess the toxic effects of sorafenib and acetaminophen on hepatocytes [22]. Although the above liver organ-on-a-chip featured the capability of toxicity testing, the entire chip was treated as a whole unit subject to analysis, resulting in the poor parallelism of analytical testing conducted on a single chip. Digital microfluidics technology, as a new type of liquid processing technology, combines droplets and microfluidics technology to form multiple analysis units in parallel on a single chip [23, 24]. Each droplet is taken as an independent analysis unit, which increases the parallelism of analytical testing and achieves more accurate and sensitive results than the traditional analog analysis of organ-on-a-chip [25]. Au et al. prepared a liver-on-a-chip platform via digital microfluidic technology to evaluate individual 3D liver organoids' responsiveness to drugs
[26]. Nevertheless, traditional digital microfluidics platforms can only perform multiplex or parallel biochemical reactions due to the use of a small number of droplets (from several up to tens) [27]. In addition, traditional digital microfluidics platforms have not been leveraged for 3D cell culture with continuous perfusion [28]. Therefore, the development of an advanced digital organ-on-a-chip platform with good parallelism, simple preparation and operation would be a significant step in the screening of anticancer drugs and toxicity testing, enabling a more efficient drug screening pipeline for liver cancer.

In this study, we developed a novel digital organ-on-achip platform, on which uniform hydrogel microspheres containing liver cancer cells, human vascular endothelial cells and human fibroblasts were well separated via microwells to improve parallel analysis and reduce variation caused by the "single pot" analysis in traditional organon-a-chip. Although cell microspheres can be generated via gravity or self-assembled on-chip [29-31], the size and shape of cell spheres are not well-controlled, which may lead to unwanted variation in subsequent biological analysis [32]. The cell microspheres generated through water-in-oil-based microfluidics are generally contaminated with oil, which can also cause bias in drug testing $[33,34]$. In this study, uniform cellular microspheres with a diameter of $200 \mu \mathrm{m}$ were prepared via electrospray, and they served as individual analysis units in this digital organ-on-a-chip. The uniform microsphere and microwell array divided the traditional "single pot" analysis unit into hundreds of independent analysis units, which was therefore characterized by high parallelism. The microwell array immobilized the microspheres under the condition of perfusion and provided a relatively independent space for growth and functioning. In addition, due to the design of our microwell array, liver cancer microspheres immobilized in each well were adequately separated, allowing for digital fluorescence analysis without the interference of adjacent fluorescence signals. Our results showed that, at a concentration of $10 \mu \mathrm{M}$, sorafenib demonstrated a clear cytotoxic effect on liver cancer cells in the digital organ-on-a-chip, which was consistent with the results previously reported in vivo [35]. Furthermore, our digital platform demonstrated the feasibility of developing natural killer cell-derived extracellular vesicle (NK EV) based anticancer therapeutics, providing an opportunity for the evaluation and developmental acceleration of novel therapeutic platforms, such as biologics, functional materials and EVs. Therefore, the proposed digital organ-on-a-chip based on high parallelism and uniform analysis units provides a robust platform for cancer drug development and toxicity screening for drug candidates for cancer and other diseases, and is expected to expedite the process of drug development in vitro. 


\section{Materials and methods}

\section{Design and fabrication of digital and traditional organ-on-a-chip}

The bottom layer of the novel digital organ-on-a-chip consisted of 127 circular holes with a diameter of $300 \mu \mathrm{m}$ and a depth of $200 \mu \mathrm{m}$ arranged in a regular hexagonal microarray. The interval between adjacent circular holes was $150 \mu \mathrm{m}$. In contrast, the bottom layer of the traditional "single pot" organ-on-a-chip was a regular hexagonal cavity ( side length $=2.7 \mathrm{~mm}$, depth $=200 \mu \mathrm{m}$ ). The top layer of both types of organ-on-a-chip contained channels and ports for dynamic perfusion, and a regular hexagonal chamber (side length $=2.7 \mathrm{~mm}$, depth $=1 \mathrm{~mm}$ ) matching the bottom layer.

For the device fabrication process, polydimethylsiloxane (PDMS) prepolymer and curing agent (10:1 ratio, Dow Corning, Midland, MI, USA) were mixed and uniformly stirred, then poured on the mold and cured at $80{ }^{\circ} \mathrm{C}$ for $4 \mathrm{~h}$. The PDMS was subsequently peeled off and the excess was appropriately cut off. The bottom and top layers were then bonded in a vacuum plasma machine (Putele, Suzhou, China). The chamber surface was modified by Pluronic F-127 [36] (10\% w/v, Sigma-Aldrich, St. Louis, Missouri, USA) for $2 \mathrm{~h}$ and rinsed using PBS $(0.01 \mathrm{M})$ for $10 \mathrm{~min}$. The contact angle of the bottom surfaces after Pluronic F-127 treatment was measured using a drop shape analyzer (DSA100, KRÜSS, Germany).

\section{Construction of liver cancer microspheres}

\section{Cell transduction}

Liver cancer cells (HepG2) and human umbilical vein endothelial cells (HUVEC) were provided by Prof. Ben Wang's laboratory (Zhejiang University, Zhejiang, China), and human foreskin fibroblasts (HFF-1) were obtained from the China Center for Type Culture Collection (CCTCC). The HepG2, HUVEC and HFF-1 cells were seeded at $5 \times 10^{5}$ cells per well in 24 -well plates and cultured in Dulbecco's Modified Eagle Medium (DMEM) containing 10\% fetal bovine serum (Gibco, Melbourne, Australia) in an incubator $\left(5 \% \mathrm{CO}_{2}\right)$ at $37{ }^{\circ} \mathrm{C}$. The HepG2, HUVEC and HFF-1 cells were transduced at about $80 \%$ confluency with lentiviruses of pLenti-CMV-mCherry3FLAG-PGK-Puro (OBIO Technology, Shanghai, China), pHBLV-CMV-EF1-ZsGreen-P2A-Puro (Hanbio Biotechnology, Shanghai, China) and pSLenti-EF1a-EBFP2P2A-Puro-CMV-HA-MCS (OBIO Technology, Shanghai, China), respectively. After $72 \mathrm{~h}$ of transduction, the target cells were observed under a fluorescence microscope to confirm that the lentiviruses were successfully transfected into the target cells, and puromycin-containing medium (MedChem Express, Monmouth Junction, NJ, USA) was added for the selection of resistant colonies.

\section{Generation of microspheres via microfluidic electrospray}

HepG2, HUVEC, and HFF-1 cells were mixed at a ratio of $4: 1: 4$ in $2 \%(\mathrm{w} / \mathrm{v})$ sodium alginate solution with a total cell concentration of $10^{6}$ cells $/ \mathrm{mL}$, and dispensed using a highvoltage electrostatic field system to generate uniform cellular microspheres [37]. The microspheres' diameter was controlled by adjusting electrostatic field voltage, pump rate, and other parameters. The microspheres were stable in $2 \% \mathrm{CaCl}_{2}$ solution and washed with culture medium three times for prior culturing on a digital organ-on-a-chip.

\section{Simulation of chemical diffusion in traditional and digital organ-on-a-chip}

The molecular weight (MW) of sorafenib is 464.8 , which is close to the MW of glucose (180). The MW of interferon- $\gamma$ $(\mathrm{IFN}-\gamma)$ in NK extracellular vesicle is $1.7 \times 10^{4}$, which is close to the MW of $\alpha$-lactalbumin $\left(1.4 \times 10^{4}\right)$. Thus, for the numerical simulation, the diffusion coefficients of glucose and $\alpha$-lactalbumin in $2 \%$ sodium alginate $\left(6.4 \times 10^{-10} \mathrm{~m}^{2} / \mathrm{s}\right.$, $\left.1.0 \times 10^{-10} \mathrm{~m}^{2} / \mathrm{s}\right)[38,39]$ were used for sorafenib and IFN- $\gamma$ in $2 \%$ sodium alginate, respectively. The convection-diffusion equation was employed to quantitatively analyze the diffusion speed of both small (Sorafenib, MW $<10^{4}$ ) and large molecules (IFN- $\gamma$ ) in the traditional "single pot" organ chip and digital organ-on-a-chip.

Diffusion of chemicals in the traditional organ-on-a-chip:

Governing equation:

$\frac{\partial C(x, t)}{\partial t}=D \frac{\partial^{2} C(x, t)}{\partial x^{2}}$.

Initial condition:

$C(x, 0)= \begin{cases}0, & x \neq l, \\ C_{0}, & x=l .\end{cases}$

Boundary conditions:

$\begin{cases}\frac{\partial C(x, t)}{\partial x}=0, & x=0, \\ C(x, t)=C_{0}, & x=l .\end{cases}$

Diffusion of chemicals in digital organ-on-a-chip:

Governing equation:

$\frac{\partial C(x, t)}{\partial t}=\frac{D}{x^{2}} \frac{\partial}{\partial x}\left(x^{2} \frac{\partial C(x, t)}{\partial x}\right)$. 
Initial condition:

$C(x, 0)= \begin{cases}0, & x \neq r, \\ C_{0}, & x=r .\end{cases}$

Boundary conditions:

$\begin{cases}\frac{\partial C(x, t)}{\partial x}=0, & x=0, \\ C(x, t)=C_{0}, & x=r .\end{cases}$

Here, $\partial$ denotes partial derivative; $C(x, t)$ denotes the chemical concentration compound; the initial concentrations of sorafenib and IFN- $\gamma\left(C_{0}\right)$ were $10 \mu \mathrm{mol} / \mathrm{L}$ and $50 \mu \mathrm{g} / \mathrm{mL}$, respectively; $x$ denotes the distance from the chip surface to its bottom; $t$ denotes the time; $l$ denotes the diffusion distance of substances in the traditional "single pot" chip, which was $200 \mu \mathrm{m} ; r$ denotes the diffusion distance of substances in the digital organ-on-a-chip, which was $100 \mu \mathrm{m}$; the diffusion coefficients $(D)$ of sorafenib and IFN- $\gamma$ in $2 \%$ sodium alginate were $6.4 \times 10^{-10} \mathrm{~m}^{2} / \mathrm{s}$ and $1.0 \times 10^{-10} \mathrm{~m}^{2} / \mathrm{s}$, respectively. The MATLAB software (2017a, MathWorks) was used to solve the convective-diffusion equation.

\section{Characterization of liver cancer microspheres in digital organ-on-a-chip}

Before loading cellular microspheres onto the digital organ-ona-chip, the channels were perfused with $2 \%$ penicillin-streptomycin (Biological Industries, Beit Haemek, Israel) for $2 \mathrm{~h}$. The microsphere suspension was infused into a digital organon-a-chip using a peristaltic pump (Longer Pump, Baoding, China), and allowed to stand for $15 \mathrm{~min}$. Subsequently, the medium was continually perfused at a flow rate of $0.25 \mathrm{~mL} /$ min. The viability of liver cancer microspheres on days 1,3 , 7 and 14 in the chips was analyzed via live/dead cell staining (Dojindo, Kumamoto, Japan). In brief, Calcein-AM (2 $\mu \mathrm{L})$ and propidium iodide $(3 \mu \mathrm{L})$ were added to DMEM $(1 \mathrm{~mL})$ to prepare a staining solution. The microspheres were then incubated with the staining solution for $30 \mathrm{~min}$ in the dark; they were imaged under a Leica TCS SP confocal microscope (Leica, Nussloch, Germany) and analyzed with Image $\mathrm{J}$ software. For the immunofluorescence assay, liver cancer microspheres were fixed with $4 \%$ paraformaldehyde and permeabilized with $1 \%$ Triton X-100 solution. Afterward, the microspheres were blocked in $1 \%$ bovine serum albumin (BSA) solution (Sigma, St. Louis, MO, USA) for $1 \mathrm{~h}$. Next, some microspheres were incubated together with rabbit antiCD31 antibody (1:500, Abcam, Cambridge, UK) and chicken anti-albumin antibody (1:100, Abcam, Cambridge, UK) overnight at $4{ }^{\circ} \mathrm{C}$. These microspheres were then sequentially incubated with 1:1000 anti-rabbit secondary antibody labeled with Alexa Fluor 488 (Abcam, Cambridge, UK) and anti-chicken secondary antibody labeled with Alexa Fluor 594 (Abcam, Cambridge, UK) for $2 \mathrm{~h}$ each. The rest of microspheres were incubated with mouse anti-vimentin antibody (1:500, Abcam, Cambridge, UK) overnight at $4{ }^{\circ} \mathrm{C}$ and then incubated with anti-mouse secondary antibody labeled with Alexa Fluor 488 (Abcam, Cambridge, UK) for $2 \mathrm{~h}$. The nuclei were stained with 4',6-diamidino-2-phenylindole (DAPI, Beyotime, Shanghai, China). The images were observed under a Leica TCS SP confocal microscope (Leica, Nussloch, Germany).

The secretion of albumin and urea from liver cancer microspheres was measured for functional evaluation. The culture medium was collected on days 1, 3, 7 and 14 after microspheres were seeded on a digital organ-on-a-chip. The analysis was performed using the albumin and urea ELISA quantitative detection kit (Fankew, Shanghai, China) according to the manufacturers' recommendations. Absorbance was measured employing a SpectraMax M5 (Molecular Devices, San Jose, USA) multifunctional microplate reader.

\section{Evaluation of toxicity on 2D culture, traditional and digital organ-on-a-chip}

Toxicity testing using sorafenib was conducted on a 2D monolayer coculture, traditional organ-on-a-chip and digital organ-on-a-chip. The cell suspensions were mixed according to previous reports with the ratio of HepG2: HUVEC: HFF-1 set to $4: 1: 4$ ( $\times 10^{6}$ cells $)$ [40]. For the 2D monolayer coculture, the cell suspension was seeded into 24-well plates at a density of $3 \times 10^{4}$ cells per well. For the preparation of traditional organ-on-a-chip for toxicity testing against sorafenib, the cell suspension was mixed with $2 \%$ sodium alginate solution as described above, and seeded into a "single pot" hexagonal microfluidic device with a side length of $2.7 \mathrm{~mm}$ and a depth of $200 \mu \mathrm{m}$. The digital organ-on-a-chip device was prepared as described above.

The 24-well plate, traditional organ-on-a-chip, or digital organ-on-a-chip were placed in an incubator $\left(37{ }^{\circ} \mathrm{C}\right.$, $5 \% \mathrm{CO}_{2}$ ) for $72 \mathrm{~h}$. Among them, the traditional and digital organ-on-a-chip were cultured with medium perfusion at a rate of $0.25 \mathrm{~mL} / \mathrm{min}$. On day 4 , cells cultured under these three conditions were continuously exposed to $0.0,5.0,10.0$, or 20.0 $\mu \mathrm{M}$ sorafenib (MedChem Express, Monmouth Junction, NJ, USA) solution for $48 \mathrm{~h}$ for drug toxicity testing. According to the configuration of live/dead cell staining, the proportion of live cells (green) in the confocal images was measured using Image $\mathbf{J}$ software to obtain the cell viability. Live/dead cell staining was also performed to assess the toxic effects of sorafenib on cells under these different culture conditions.

\section{Isolation, quantification and characterization of EVs from NK-92MI cells}

NK-92MI cells were obtained from Prof. Yibing Xu's laboratory (Zhejiang University, Zhejiang, China) and cultured 
in RPMI-1640 medium (Gibco, Melbourne, Australia) supplemented with $10 \%$ fetal bovine serum (FBS, Gibco, Melbourne, Australia), and penicillin-streptomycin (1\%, Sangon Biotech, Shanghai, China). NK-92MI cell-derived EVs were isolated from the culture medium as previously reported using an ultracentrifugation method [41]. First, live cells in the medium were removed at $200 \times g$ ( $5 \mathrm{~min}$ ). Then, dead cells in the supernatant were separated at $2000 \times g$ (15 min). Subsequently, cell debris was removed through centrifugation at $12,000 \mathrm{~g}$ (45 min). A $0.2 \mu \mathrm{m}$ filter (Millipore, Billerica, MA, USA) was used to separate the obtained supernatant, which was then centrifuged at $120,000 \times g$ for 70 min to obtain EVs pellets. Pellets were washed and resuspended with RNAse inhibitor-containing DPBS (1 U/ $\mu \mathrm{L}$, Gibco, Gaithersburg, USA). The protein concentration of EVs was quantified using a bicinchoninic acid (BCA) kit (Sangon Biotech, Shanghai, China).

\section{Nanoparticle tracking analysis (NTA)}

The size distributions of EV samples were measured with a NanoSight NS300 (Malvern Instruments, Malvern, UK) [41]. NTA 3.0 software was used to control the instrument. Five videos with $30 \mathrm{~s}$ each were recorded for each measurement. The screen gain, camera level and detection threshold were set to $1.0,15$ and 5 , respectively. The camera type was sCMOS.

\section{Transmission electron microscope (TEM)}

After ultracentrifugation, the EVs were fixed in $2.5 \%$ glutaric dialdehyde for $30 \mathrm{~min}$, washed 3 times with PBS, then resuspended in $0.5 \% \mathrm{BSA} / \mathrm{PBS}$. Twenty microliters of EV solution were placed on each microscope grid. A filter paper was applied to absorb the redundant solution, and then the grid was exposed in a dry environment for $5 \mathrm{~min}$. Subsequently, the EVs were stained with uranyl acetate for $5 \mathrm{~min}$, and the samples were visualized using a JEM-1010 transmission electron microscope ( $80 \mathrm{kV}$, JEOL, Tokyo, Japan).

\section{Western blot (WB)}

The identification of Calnexin, TSG101, HSC70 and CD63 in both NK-92MI cells and NK-92MI-derived EVs was confirmed via WB [42]. Briefly, NK-92MI cells $(20 \mu \mathrm{g})$ and EVs lysates $(50 \mu \mathrm{g})$ were separated using sodium dodecyl sulfate (SDS)-gel electrophoresis, and they were subsequently transferred to a polyvinylidene fluoride membrane. The membrane was then blocked by $5 \%$ skimmed milk in tris-buffered saline (TBS) for $2 \mathrm{~h}$, incubated with rabbit anti-calnexin antibody (1:1000, Abcam, Shanghai, China), rabbit anti-TSG101 antibody (1:500, Abcam, Shanghai, China), rabbit anti-HSC70 antibody (1:1000,
Abcam, Shanghai, China), or rabbit anti-CD63 antibody (1:1000, Abcam, Shanghai, China) at $4{ }^{\circ} \mathrm{C}$ overnight, and then washed 3 times using Tris-buffered saline with $0.1 \%$ Tween 20 (TBST) for 10 min. The membrane was further incubated with goat anti-rabbit immunoglobulin G (IGg) H\&L (horseradish peroxidase, HRP) secondary antibody $(1: 1000)$ at room temperature for $2 \mathrm{~h}$. The membrane was incubated with TBST for $10 \mathrm{~min}$, and the procedure was repeated 3 times prior to analysis on a Bio-Rad chemiluminescence imaging system (Hercules, California, USA).

\section{Therapeutic effect of NK EVs on liver cancer cells in digital organ-on-a-chip}

The lethal effect of NK-derived EVs on liver cancer was tested using digital organ-on-a-chip. First, liver cancer microspheres were placed in a digital organ-on-a-chip and incubated for $48 \mathrm{~h}\left(37{ }^{\circ} \mathrm{C}, 5 \% \mathrm{CO}_{2}\right)$. Then, NK-derived EVs were continuously perfused at concentrations of 0 , $10.0,30.0$, and $50.0 \mu \mathrm{g} / \mathrm{mL}$ for $24 \mathrm{~h}$. The cell viability was evaluated via live/dead cell staining under a Nikon confocal microscope with the aid of Image J software.

\section{Statistical analysis}

All statistical analyses were performed using the software GraphPad version 6.0. Data were presented as mean \pm standard deviation (SD). $p$ values $<0.05$ were considered as statistically significant.

\section{Results}

\section{Characterization of digital organ-on-a-chip}

The digital organ-on-a-chip was designed to contain two layers of PDMS: the top layer consisting of a hexagon chamber matching the layout of the bottom chamber, and the bottom chamber embedded with an array of circular wells in a hexagon shape (Fig. 1a). Following the hydrophilic treatment of the microfluidic channels and hexagon chamber with Pluronic F-127 (10\% v/v) (Fig. 1b), the contact angle was $11.7^{\circ} \pm 0.9^{\circ}$ as opposed 10 to $109.9^{\circ} \pm 9.4^{\circ}$ in the untreated control (Figs. 1bI-1bIII). The hydrophilic treatment increased the capture rate of cellular microspheres in the hexagon microarray to $(75.4 \pm 3.6) \%$ at a flow rate of $0.5 \mathrm{~mL} / \mathrm{min}$ compared to $(8.9 \pm 2.1) \%$ $(p<0.05)$ in the untreated control (Fig. 1bIV). Representative images of liver cancer microspheres in the digital organ-on-a-chip are shown in Fig. 1c. 
Fig. 1 Development of a digital organ-on-a-chip device. a (I, II) 3D schematic diagram of digital organ-on-a-chip (top layer and microchannel, bottom layer, and microwell array) and (III) physical image. b (I-III) Comparison of water contact angle and (IV) capture rate of microspheres in the digital organ-on-a-chip before and after treatment with Pluronic F-127. c (I) 3D schematic diagram; (II, III) physical image and enlarged view of microspheres in the digital organ-on-a-chip. Scale bar: $500 \mu \mathrm{m}(\mathrm{c}-\mathrm{II})$. Scale bar: $50 \mu \mathrm{m}(\mathbf{c}-\mathrm{III})$. The data were averaged from three samples and expressed as mean $\pm \mathrm{SD}$ $\left({ }^{*} p<0.05\right.$, NS, $p>0.05$ nonsignificant) (a) (I)
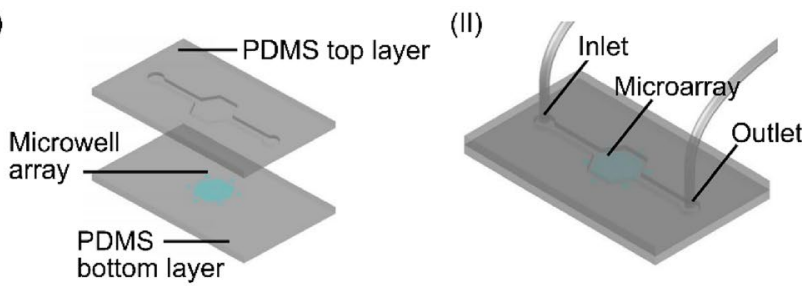

(II)

(b) (I)
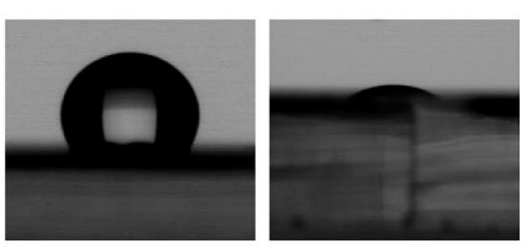

(III)

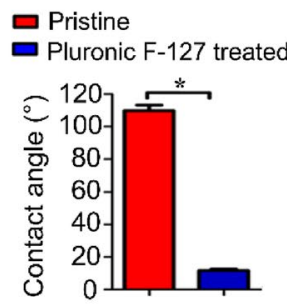

- Pluronic F-127 treated

(IV) $\square$ Pristine

- Pluronic F-127 treated

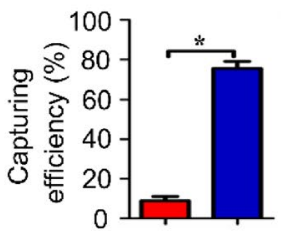

(c) (I)

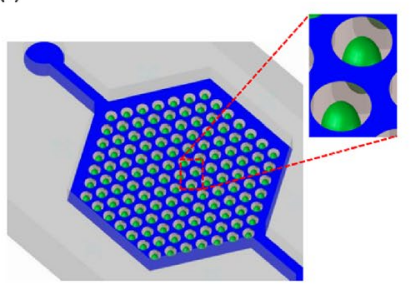

(II)

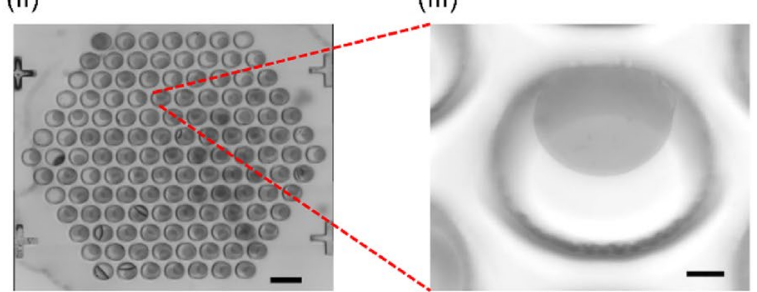

\section{Preparation of uniform liver cancer microspheres}

The liver cancer microspheres prepared via electrospray microfluidic technology were obtained with reduced labor and time consumption [43]. The prepared sodium alginate microspheres containing no cells appeared to be bright and transparent under a bright-field microscope (Figs. 2a-2c). In comparison, sodium alginate microspheres containing HepG2, HUVEC, and HFF-1 cells appeared to be evenly dotted due to the presence of cellular clusters (Figs. 2d-2f). Both types of sodium alginate microspheres had a relatively uniform circular shape. By controlling the voltage and flow rate, sodium alginate microspheres could be generated with a diameter of 160,250 , or $360 \mu \mathrm{m}$ on a large scale with normal Pearson distribution (Figs. 2g-2i). We selected cellular microspheres with a uniform diameter of $200 \mu \mathrm{m}$ containing HepG2, HUVEC and HFF-1 cells and placed them in the digital organ-on-a-chip for subsequent experiments.

\section{Modeling of molecular diffusion in traditional and digital organ-on-a-chip}

Via establishing a computational model, the diffusion rate of a small molecule in the traditional "single pot" organon-a-chip and the digital organ-on-a-chip was calculated by taking $10 \mu \mathrm{M}$ sorafenib solution (diffusion coefficient: $6.4 \times 10^{-10} \mathrm{~m}^{2} / \mathrm{s}$ ) and $50 \mu \mathrm{g} / \mathrm{mL}$ NK cell EVs solution (diffusion coefficient: $1.0 \times 10^{-10} \mathrm{~m}^{2} / \mathrm{s}$ ) as examples. In the case of a small compound (e.g., sorafenib), the concentration on the surface and in the center of sodium alginate microspheres reached steady-state after $17.3 \mathrm{~s}$ in the digital organ-on-achip. After $215.9 \mathrm{~s}$, sorafenib on the top surface or at the bottom of hexagonal sodium alginate gel in the traditional "single pot" organ-on-a-chip reached the same concentration (Fig. 3a). In the case of large molecules such as TNF- $\alpha$ and IFN- $\gamma$, the diffusion efficiency was also significantly slowed down. More specifically, it would require $94.7 \mathrm{~s}$ for these large molecules to reach the state of homeostasis of diffusion into and out of sodium alginate microspheres in the digital organ-on-a-chip. In comparison, the concentration of large molecules would need $1594.3 \mathrm{~s}$ to stabilize throughout the hexagonal sodium alginate gel in the traditional "single pot" organ-on-a-chip (Fig. 3b). Since EVs are approximately $100 \mathrm{~nm}$ in diameter and they encapsulate many types of large molecules such as TNF- $\alpha$ and IFN- $\gamma$, it is reasonable to speculate that $\mathrm{EVs}$ require more time to enter microspheres than drugs such as sorafenib.

\section{Long-term culture of cellular microspheres in digital organ-on-a-chip}

In order to observe the growth of HepG2, HUVEC and HFF-1 cells in the sodium alginate microspheres, lentiviruscarried red, green, and blue fluorescent proteins were transferred to the HepG2, HUVEC, and HFF-1 cells, respectively (Fig. 4a). The immunofluorescence staining of liver cancer 
Fig. 2 Uniform microspheres were prepared via microfluidic electrospray. a-c Brightfield microscope images of different sizes of $2 \%$ sodium alginate microspheres without cells. d-f Brightfield microscope images of different sizes of $2 \%$ sodium alginate microspheres encapsulating HepG2, HUVEC and HFF-1 cells. $\mathbf{g}-\mathbf{i}$ Diameter distribution of liver cancer microspheres, with 50 microspheres measured in each figure. Scale bar, $100 \mu \mathrm{m}(\mathbf{a}-\mathbf{f})$ (a)

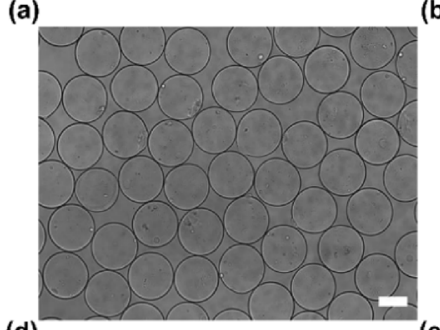

(d)

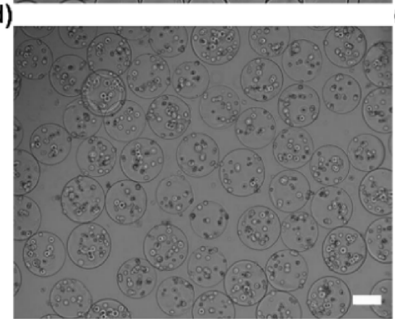

(g)

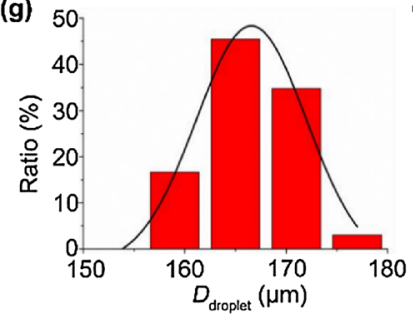

(b)

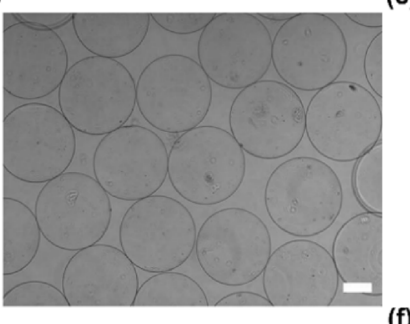

(e)

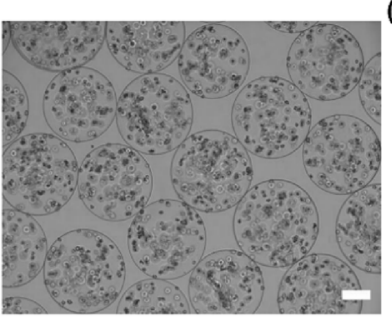

(h) 50

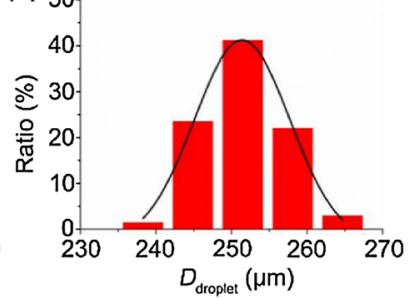

(c)

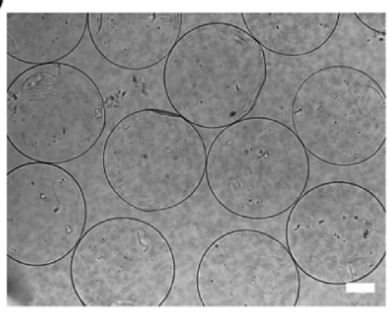

(f)

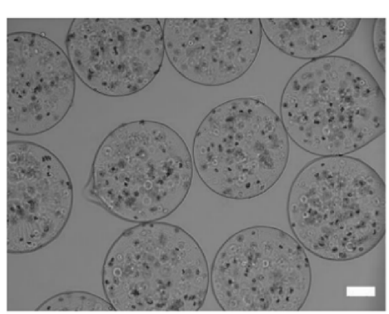

(i) 50

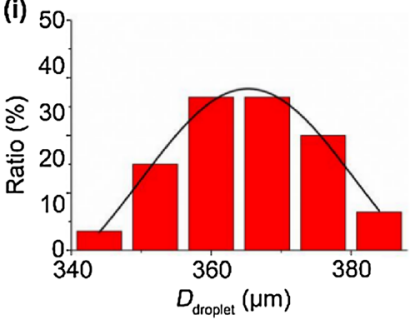

(a) Traditional organ-on-a-chip
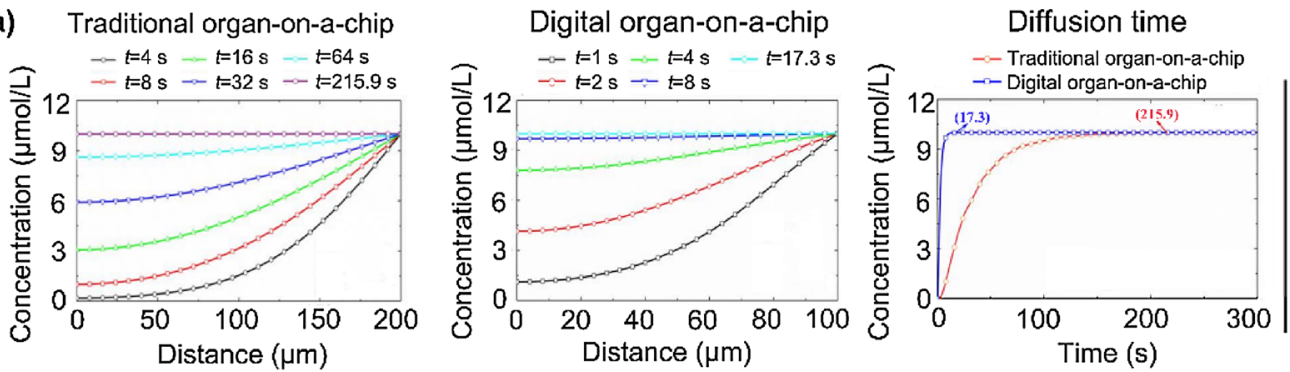

Small

molecules

(b)
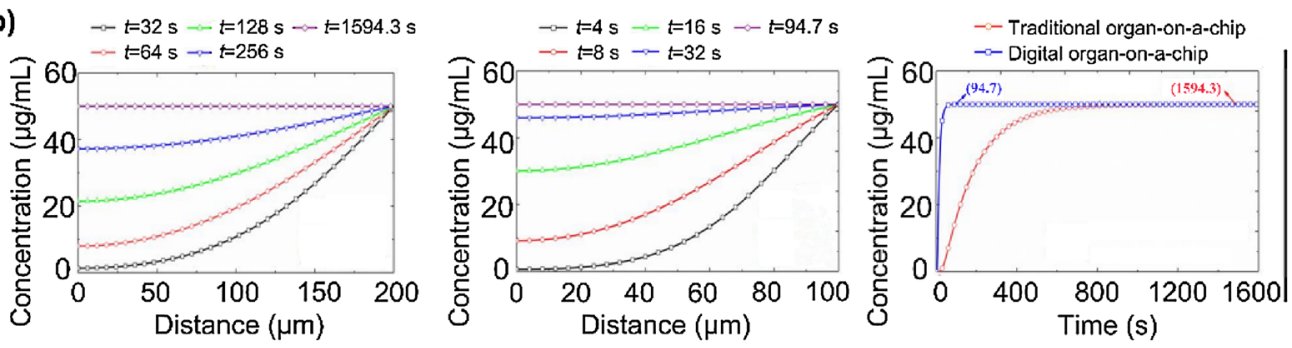

Large

molecules

Fig. 3 The diffusion rates of therapeutic compounds with different molecular weights in traditional "single pot" organ-on-a-chip and digital organ-on-a-chip were simulated by a convection-diffusion equation. In the traditional "single pot" organ-on-a-chip, the compound needed to diffuse from the surface of the hydrogel to the bottom, and the diffusion distance was $200 \mu \mathrm{m}$. In the digital organ-ona-chip, it only needed to diffuse from the surface of the microsphere to the center, and the diffusion distance was $100 \mu \mathrm{m}$. a is the diffusion rate of relatively small molecular compounds (e.g., sorafenib, $\mathrm{MW}=464.8$ ) in traditional "single pot" organ-on-a-chip and digital organ-on-a-chip, and $\mathbf{b}$ is the diffusion rate of relatively large molecules (e.g., IFN- $\gamma, \mathrm{MW}=1.7 \times 10^{4}$ ) in traditional "single pot" organon-a-chip and digital organ-on-a-chip 
Fig. 4 Characterization and function of cellular microspheres in the digital organon-a-chip. a Representative inverted fluorescence microscope images of transduced HepG2 cells, HFF-1 cells and HUVEC cells in sodium alginate microspheres. b In the microspheres cultured for 5 days, Vim protein expressed green fluorescence, CD31 protein, ALB protein expressed red fluorescence, and nuclei expressed blue fluorescence. c Live/dead cell-stained confocal images of liver cancer microspheres cultured on the digital organ-on-a-chip for 1, 3, 7 , and 14 days. $\mathbf{d}$ Cell viability analysis on day $1,3,7$, and 14 $(n=3)$. e and $\mathbf{f}$ Measurements of albumin and urea secreted by liver cancer microspheres in the digital organ-on-a-chip. The data were averaged from three samples and expressed as mean $\pm \mathrm{SD}(* p<0.05, \mathrm{NS}$, $p>0.05$ nonsignificant). Scale bar: $100 \mu \mathrm{m}(\mathbf{a}), 50 \mu \mathrm{m}(\mathbf{b})$, $200 \mu \mathrm{m}(\mathbf{c})$ (a)
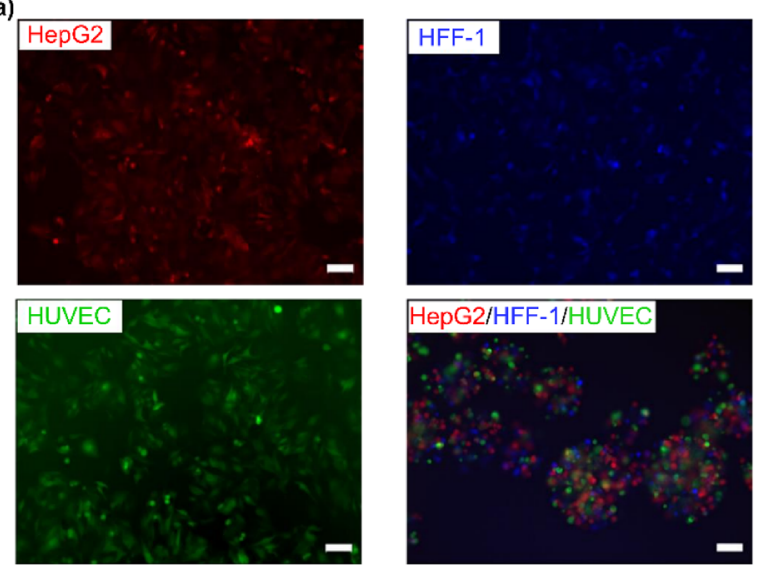

(b)

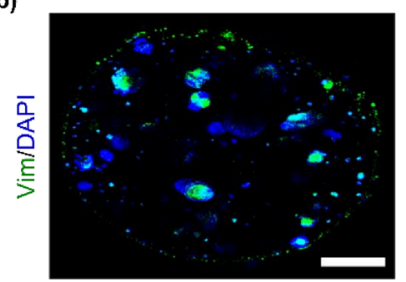

(c)
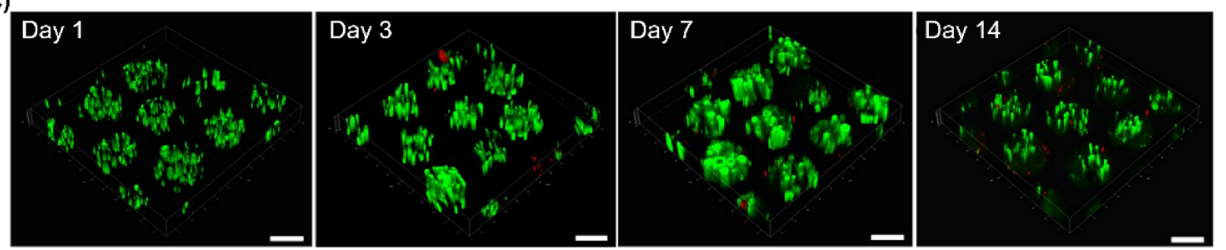

(d)

(e)
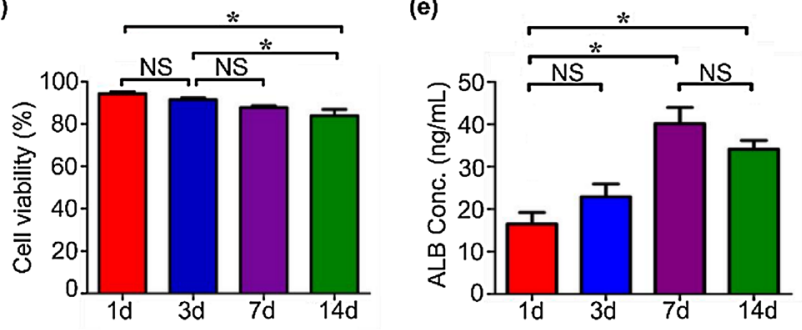

(f)
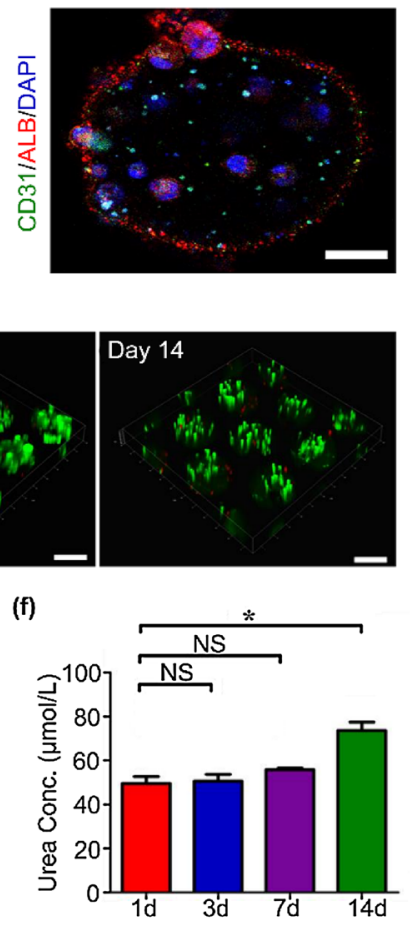

showed that HepG2-related protein ALB (red), HUVECrelated protein CD31 (green), and HFF-1-related protein vimentin (Vim, Green) were expressed in cellular microspheres (Fig. 4b) on the 5th day of microsphere culture onchip. The cell viability of cellular microspheres in the digital organ-on-a chip on day $1,3,7$ or 14 was $(94.3 \pm 1.0) \%$, $(91.5 \pm 0.9) \%,(87.7 \pm 0.9) \%$, or $(83.8 \pm 3.1) \%$, respectively (Figs. 4c and 4d). Although the cell viability decreased slightly on day 7 and 14, it showed no significant change between day 3 and day $7(p>0.05)$, whereas the change was significant between day 3 and day $14(p<0.05)$. The overall survival rate of cellular microspheres remained above $80 \%$ during the 14-day culture period despite a significant drop when comparing day 14 with day 1 (Figs. 4c and 4d).

The functional activity of cellular microspheres was evaluated by measuring the contents of albumin and urea in the culture medium (Figs. 4e and 4f). The secretion of albumin from microspheres on day $1,3,7$, and 14 was $16.5 \pm 2.7$, $22.9 \pm 2.9,40.1 \pm 3.8$, and $34.1 \pm 2.1 \mathrm{ng} / \mathrm{mL}$, respectively. There was no significant difference in albumin levels between day 1 and 3, whereas a significant increase in albumin concentration was observed on day 7 and 14 as compared to day $1(p<0.05)$. Although the secretion of albumin on day 14 was slightly less than that on day 7 , the difference was not statistically significant $(p>0.05)$ (Fig. 4e). In addition, the secretion of urea from cellular microspheres on day 1,3 , and 7,14 was $49.5 \pm 3.2,50.6 \pm 3.1,55.8 \pm 0.7$, and $73.6 \pm 3.9 \mu \mathrm{mol} / \mathrm{L}$, respectively. Different to the pattern of albumin secretion, only the concentration of urea on day 14 was significantly increased $(p<0.05)$ as opposed to day 1, 3, and 7 (Fig. 4f).

\section{Evaluation of toxicity on 2D culture, traditional and digital organ-on-a-chip}

In order to assess the toxicity testing capacities of $2 \mathrm{D}$ culture, traditional and digital organ-on-a-chip, increased dosage of sorafenib was added before the measurement of cell viability (Fig. 5). The overlapping confocal images of live/dead cell staining showed that, after $48 \mathrm{~h}$ of sorafenib treatment at 0.0 , $5.0,10.0$, and $20.0 \mu \mathrm{M}$, the cell viability in $2 \mathrm{D}$ culture was $(99.8 \pm 10.1) \%,(42.2 \pm 6.8) \%,(28.8 \pm 2.2) \%$, and $(4.4 \pm 1.9) \%$, respectively. The cell viability in the traditional organ-on-achip after $48 \mathrm{~h}$ of sorafenib treatment at $0.0,5.0,10.0$, and $20.0 \mu \mathrm{M}$ was $(95.9 \pm 4.7) \%,(95.9 \pm 1.6) \%,(83.3 \pm 2.1) \%$, and $(45.8 \pm 13.2) \%$, respectively. Meanwhile, the cell viability in 
Fig. 5 Effect of sorafenib on cell survival rate under different culture conditions. The live/ dead staining showed the results of cell co-culture sorafenib $(0.0,5.0,10.0$, and $20.0 \mu \mathrm{M})$ for $48 \mathrm{~h}$ in 24-well plates (a), in traditional organ-on-a-chip (b), and digital organ-on-a-chip (c). Representative confocal microscope images were shown in $(\mathbf{a}-\mathbf{c})$, and the viability of cells in a 24-well plate, in the traditional organ-on-a-chip and in the digital organ-on-a-chip was analyzed and compared (d). Each bar represents the mean \pm SD of three samples $(* p<0.05$, NS, $p>0.05$ nonsignificant). Scale bar: $100 \mu \mathrm{m}$ (a), $500 \mu \mathrm{m}(\mathbf{b}, \mathbf{c})$ (a)
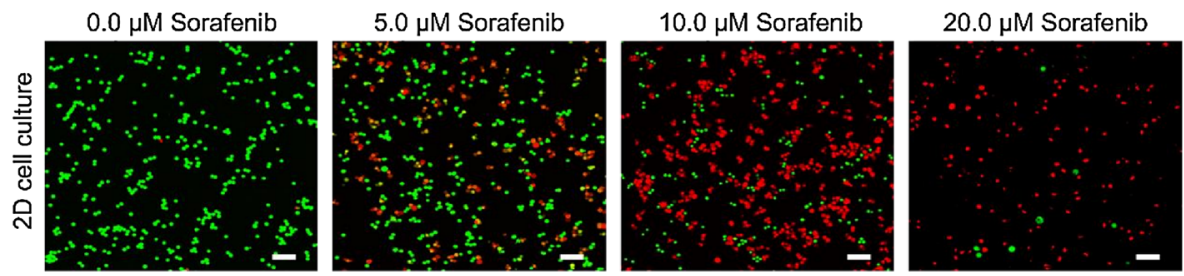

(b)
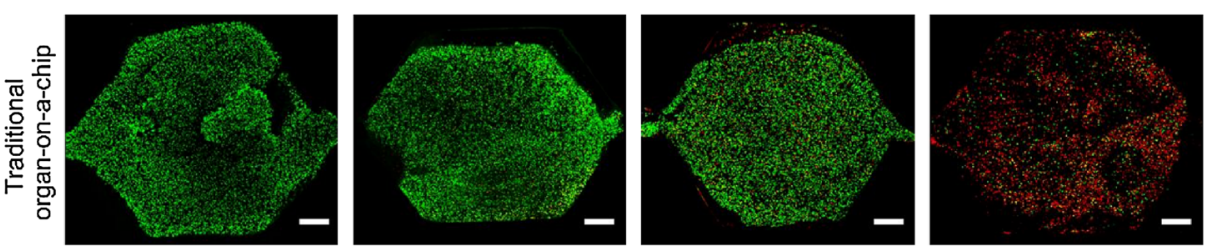

(c)
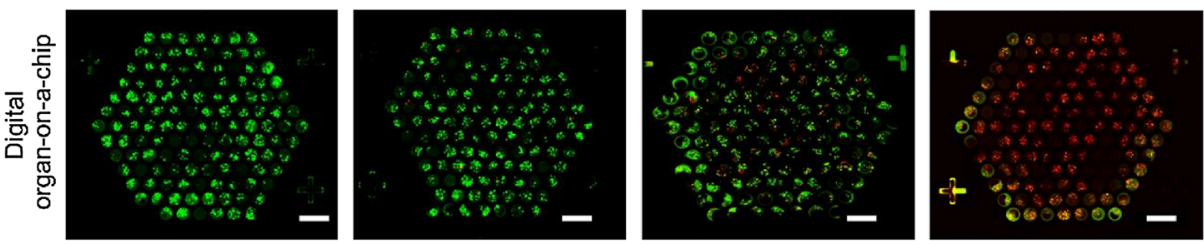

(d)

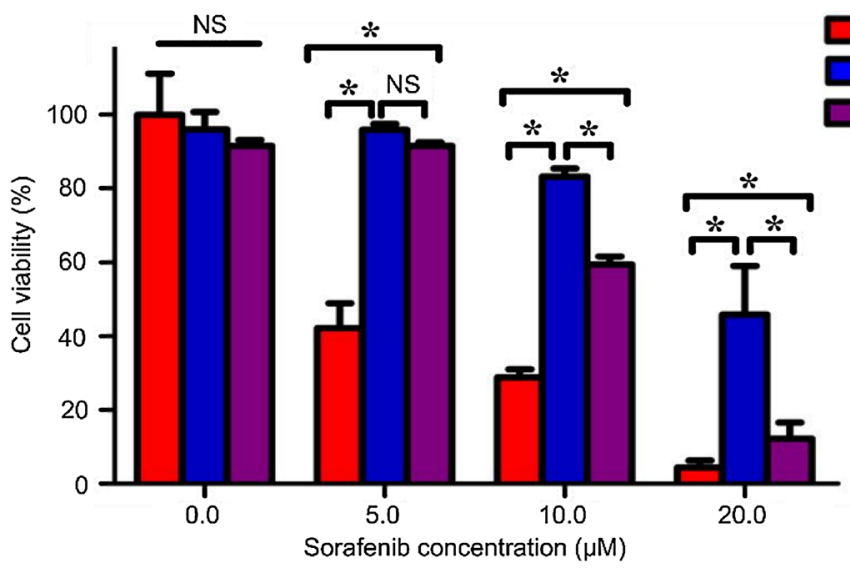

against liver cancer, EVs derived from NK-92MI cells were isolated and applied. Firstly, NK EVs were identified and characterized [44]. The peak size of NK EVs was around $100 \mathrm{~nm}$ with a non-Pearson distribution of 50-400 nm as reported by NTA (Fig. 6a). The NK EVs were round-shaped under TEM (Figs. 6b and 6c). The Western blot analysis showed that calnexin (a marker of endoplasmic reticulum) was observed in NK-92MI cells only, whereas no calnexin was detected in EVs samples. CD63 and TSG101 were both detected in EV samples, whereas HSC70 proteins existed in both cells and EV samples (Fig. 6d).

Following the administration of NK EVs into digital organ-on-a-chip for $24 \mathrm{~h}$, the cell viability of cellular microspheres treated with 10 and $30 \mu \mathrm{g} / \mathrm{mL}$ of $\mathrm{NK}$ EVs was $(96.5 \pm 2.1) \%$ and $(95.4 \pm 2.5) \%$, respectively, which was not significantly altered as opposed to the nontreatment control. In comparison, the cell viability of NK EVs treated with $50 \mu \mathrm{g} / \mathrm{mL}$ cellular microspheres was

on-a-chip for developing a new type of immune therapy 
Fig. 6 The digital organ-ona-chip was evaluated for the therapeutic effect of NK cellderived EVs on liver cancer. a The size distribution of NK cell EVs was determined by NTA. $\mathbf{b}$ and $\mathbf{c}$ Representative images of EVs in transmission electron microscope. d Western blotting confirmed the expression of exosomal markers (CD63 and TSG101) and negative markers (Calnexin). e Live/dead cell staining showed that $\mathrm{NK}$ cell EVs $(0.0,10.0,30.0,50.0 \mu \mathrm{g} /$ $\mathrm{mL}$ ) were processed with liver cancer microspheres in the digital organ-on-a-chip for $24 \mathrm{~h}$. Scale bar: $500 \mu \mathrm{m}$. Representative confocal microscope images were shown in (e). The therapeutic effects of different concentrations of EVs on cells were analyzed (f). Each bar represents the mean \pm SD of three samples $(* p<0.05$, NS, $p>0.05$ nonsignificant)

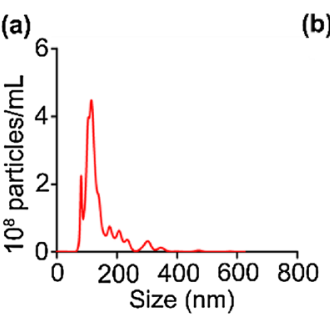

(b) (c)
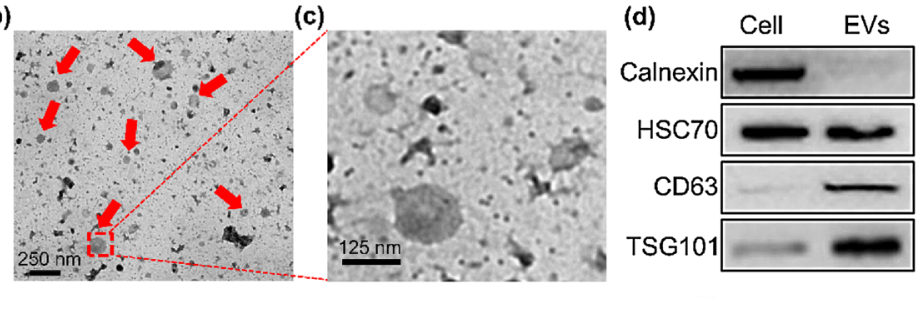

(e)

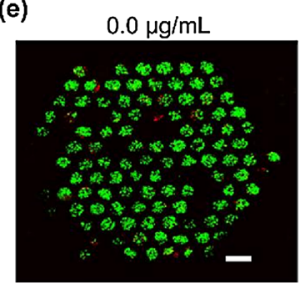

$10.0 \mu \mathrm{g} / \mathrm{mL}$

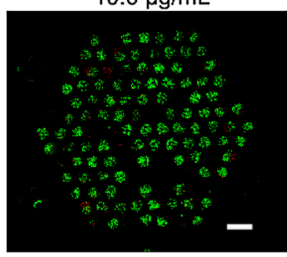

$30.0 \mu \mathrm{g} / \mathrm{mL}$
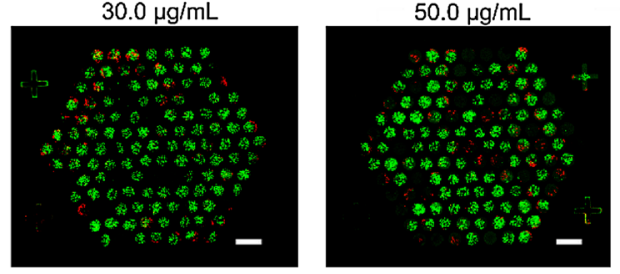

(f)

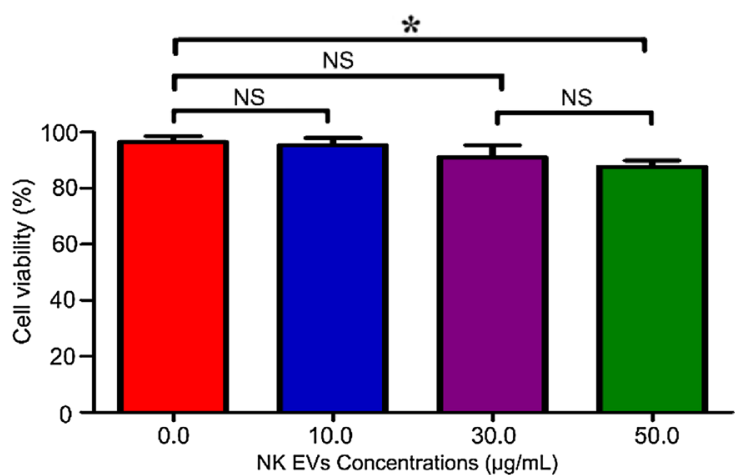

$(87.6 \pm 2.2) \%$, which was significantly lower than that of the control group ( $p<0.05$, Figs. 6e and 6f).

\section{Discussion}

Although organ-on-a-chip is a promising strategy for drug screening in vitro, the number of analysis units used for drug testing in traditional organ-on-a-chip is limited and the uniformity is poor. In this study, a novel digital organ-on-a-chip was fabricated by improving the parallelism of analysis units over the traditional organ-on-a-chip and reducing the variation. On the one hand, the design of microwell array in the digital organ-on-a-chip not only greatly increased the number of analysis units in a chip, thus improving the parallelism of the chip (Fig. 1), but also prevented the fluorescence signals between adjacent microspheres from interfering with each other. On the other hand, the uniformity of liver cancer microspheres reduced the variation of hepatotoxicity testing, and the response speed of the digital organ-on-a-chip was improved, as demonstrated by the more rapid onset of druginduced cancer cell toxicity. More importantly, the concentration of sorafenib required to kill liver cancer cells in the digital organ-on-a-chip was closer to the concentration of sorafenib killing liver cancer cells in vivo [35]. Furthermore, this chip demonstrated the feasibility of developing NK cell EV-based anti-liver cancer therapeutics; featuring high parallelism and less variation, it obviously offers a great potential for cancer drug screening and toxicity testing in vitro.

The combination of microwell array and cellular microspheres is the key not only to improving the uniformity and parallelism of digital organ-on-a-chip, but also to reducing bias in fluorescence analysis. In this type of digital organ-on-a-chip, liver cancer microspheres prepared by electrospray were of uniform size and contained a similar composition of different types of cells (Fig. S1 and Fig. 2), providing an attractive uniformity for chip analysis. Although cells can be seeded in a microarray pattern to form microspheres [45-47], the uniformity of each microsphere can be significantly affected by the initial cell seeding density, and a similar ratio of different types of cells at each spot can be even harder to achieve throughout the entire array. Herein, the analysis of 127 independent analysis units, i.e., cellular microspheres, was performed simultaneously on a single chip, which was far larger than the number previously reported in droplet-based digital organ-on-a-chip [20, 48], improving parallelism. Essentially, the distance between each microwell was $150 \mu \mathrm{m}$, which not only ensured that the liver cancer microspheres in each microwell had relatively independent growth space 
(Fig. 1c), but also minimized the fluorescence interference commonly observed in droplet-based digital organs-on-achip due to the aggregation of droplets. Thus, the proposed digital organ-on-a-chip is considered advantageous over existing droplet-based or microarray-based organ-on-achip for drug development or toxicity testing.

Our digital organ-on-a-chip provided the possibility of analysis that is closer to in vivo evaluation than the traditional "single pot" organ-on-a-chip format. As widely known, capillary vessels exist throughout the body; therefore, the diffusion distance of a drug molecule to the targeted tissue is generally within several to a few hundred of micrometers [49]. Our microspheres are the size of $200 \mu \mathrm{m}$, which is within the diffusion range of a drug molecule. In addition, the microsphere-based digital organ-on-a-chip offers a fast response that would most likely occur in vivo due to the omnipresence of capillary vessels. The computation modeling indicated that the equilibrium time of a small or large drug molecule throughout a microsphere was respectively 17.3 and $94.7 \mathrm{~s}$, which was 12.5 and 16.8 times less than single pot hydrogel (Fig. 3). The quick diffusion of drug molecules not only mimics in vivo situations, but also ensures fast and uniform responses in our digital organ-ona-chip. Thus, the microsphere-based digital organ-on-a-chip could provide an improved biomimetic situation for drug analysis over traditional "single pot" organ-on-a-chip that generally contain several millimeters of hydrogel for a drug molecule to diffuse through. Indeed, we observed that the hepatotoxic concentration of sorafenib in our digital organon-a-chip was $10 \mu \mathrm{M}$, significantly lower than that in traditional "single pot" organ-on-a-chip (Fig. 5) and closer to the concentration reported in vivo [35].

Biomimetic organoids containing multiple types of cells on-chip are essential for in vitro drug development and toxicity testing. In our digital organ-on-a-chip, we fabricated and seeded cell microspheres containing HepG2 cells, HUVEC cells and HFF-1 cells, which could more accurately represent in vivo situations over single-cell based organ-on-a-chip. As reported previously, when cells are cocultured within microspheres in a 3D microenvironment, cell-to-cell interactions help to improve the function of liver cells and better simulate the biological characteristics of liver cells in vivo [50, 51]. For example, Maharjan et al. embedded a human hepatic cell line (HepG2-C3A) and a human hepatic stellate cell line (LX-2) in gelatin methacryloyl hydrogel to prepare a 3D liver tissue model [52]. Compared with HepG2-C3A alone, the liver tissue structure formed by the co-culture of HepG2-C3A and LX-2 was closer to that in vivo, and the amount of albumin excreted by cells in co-culture was higher. Thus, our organ-on-achip platform containing multiple cell types showed more resemblance to in vivo conditions in terms of cell composition and function.
In this digital organ-on-a-chip, mechanical support provided by the use of sodium alginate hydrogel as extracellular matrix and continuous flow of medium ensured cells cultured at a magnitude of $10^{6} / \mathrm{mL}$ with high viability and functionality. Sodium alginate hydrogel can provide biomechanical microenvironment as seen in vivo for beneficial cell growth and proliferation $[53,54]$. In addition, the continuous flow of medium can provide timely removal of metabolic wastes and supply of sufficient nutrients and oxygen. In this study, we cultured cells at a concentration of $10^{6} / \mathrm{mL}$. If the concentration of cells in microspheres is too high, it can easily cause cell death due to hypoxia and nutrient deficiency; meanwhile, if the concentration of cells in microspheres is too low, cell survival and cell functions can be affected, resulting in reduced function of cellular microspheres. Previous studies have shown that a cell density of $10^{6} / \mathrm{mL}$ in sodium alginate microspheres exhibits good survival rates and functions $[55,56]$. As observed in our study, HepG2 cells preserved their viability no less than $80 \%$ over a course of 14 days and maintained biological functionality such as albumin generation and urea secretion (Figs. $4 \mathrm{c}$ and $4 \mathrm{~d}$ ). Moreover, during the 14-day culture period, the amount of albumin and urea secreted by hepatocytes increased, and these two biomarkers, to a certain degree, showed a positive correlation (Figs. 4e and 4f). Therefore, our organ-on-a-chip platform provides biomechanical extracellular matrix and timely mass exchange for favorable cell culture and functionality.

A better ability to mimic the in vivo microenvironment is of great importance to reliably predict drug efficacy and host responses in vivo, particularly for unknown therapeutic candidates, aimed at facilitating drug discovery and pipeline development. In this study, we explored the anti-liver cancer ability of NK cell-derived EVs in our digital organon-a-chip, and demonstrated the feasibility of developing NK cell EV-based anti-liver cancer therapeutics. Previous studies have found that NK cell EVs can be used to kill neuroblastoma and melanoma by releasing IFN- $\gamma$ and TNF- $\alpha$ [57-59], suggesting that NK cell EVs may comprise a potential immunotherapy platform for liver cancer. Our results showed that $50 \mu \mathrm{g} / \mathrm{mL}$ of NK EVs had a mild anticancer effect (Fig. 6), which may serve as a modest basis for evaluating their anti-liver cancer activity in vivo. Thus, the digital organ-on-a-chip containing a number of liver organoids is considered an attractive platform to accelerate the research and development of anticancer drugs and to explore new anticancer modalities.

\section{Conclusions}

In this study, we developed a novel digital organ-on-achip based on the combination of microwell array and cellular microspheres, which not only increases the 
parallelism in analysis units, but also minimizes analytical variation in fluorescence detection. Compared to "single pot" organ-on-a-chip, our digital organ-on-a-chip containing more than one hundred organoids transforms the traditional approach of holistic analysis to digital analysis and provides the biomimetic evaluation of drug response and toxicity testing in vitro. Overall, the use of cellular microspheres significantly increases the "digital" resolution of drug evaluation results on this novel organon-a-chip compared to its traditional counterpart in a "single pot" manner. We envision that this digital organon-a-chip platform can be broadly adapted by altering the composition of cells within the lung, kidney, brain, etc., to accelerate the development of anticancer drugs and to screen for the side effects of drug candidates for other diseases.

Supplementary Information The online version contains supplementary material available at https://doi.org/10.1007/s42242-022-00188-1.

Acknowledgements We acknowledge the supports from the General Program (No. 31871016), the National Key Scientific Instrument and Equipment Development Projects (No. 61827806) from the National Natural Science Foundation of China, the National Major Science and Technology Projects (No. 2018ZX10732401-003-007) and the National Key Research and Development Program (No. 2016YFC1101302) from the Ministry of Science and Technology of China, the National Natural Science Foundation of China (No. 81770719), and Science and Technology Department of Zhejiang Province (No. 2019C03029).

Author contributions SW, ZD, JS generated the concept, designed the experiment and involved in writing the manuscript. GW performed the experiments, collected data, conducted data analysis and interpretation, and wrote the manuscript. JW, ZL, SS, DW, HL, YH, and RW helped in experiments, data analysis, and statistical analysis. XW and HX contributed to computation simulation.

\section{Declarations}

Conflict of interest The authors declare that they have no conflict of interest.

Ethical approval This article does not contain any studies that required human or animal subjects.

Open Access This article is licensed under a Creative Commons Attribution 4.0 International License, which permits use, sharing, adaptation, distribution and reproduction in any medium or format, as long as you give appropriate credit to the original author(s) and the source, provide a link to the Creative Commons licence, and indicate if changes were made. The images or other third party material in this article are included in the article's Creative Commons licence, unless indicated otherwise in a credit line to the material. If material is not included in the article's Creative Commons licence and your intended use is not permitted by statutory regulation or exceeds the permitted use, you will need to obtain permission directly from the copyright holder. To view a copy of this licence, visit http://creativecommons. org/licenses/by/4.0/.

\section{References}

1. Bray F, Ferlay J, Soerjomataram I et al (2018) Global cancer statistics 2018: GLOBOCAN estimates of incidence and mortality worldwide for 36 cancers in 185 countries. CA Cancer J Clin 68(6):394-424. https://doi.org/10.3322/caac.21492

2. Faivre S, Rimassa L, Finn RS (2020) Molecular therapies for HCC: looking outside the box. J Hepatol 72(2):342-352. https:// doi.org/10.1016/j.jhep.2019.09.010

3. Esch EW, Bahinski A, Huh D (2015) Organs-on-chips at the frontiers of drug discovery. Nat Rev Drug Discov 14(4):248-260. https://doi.org/10.1038/nrd4539

4. Jo Y, Choi N, Kim K et al (2018) Chemoresistance of cancer cells: requirements of tumor microenvironment-mimicking in vitro models in anti-cancer drug development. Theranostics 8(19):5259-5275. https://doi.org/10.7150/thno.29098

5. Sood D, Tang-Schomer M, Pouli D et al (2019) 3D extracellular matrix microenvironment in bioengineered tissue models of primary pediatric and adult brain tumors. Nat Commun 10(1):4529. https://doi.org/10.1038/s41467-019-12420-1

6. Mao M, Bei HP, Lam CH et al (2020) Human-on-leaf-chip: a biomimetic vascular system integrated with chamber-specific organs. Small 16(22):2000546. https://doi.org/10.1002/smll.202000546

7. Zhong J, Yang Y, Liao L et al (2020) Matrix stiffness-regulated cellular functions under different dimensionalities. Biomater Sci 8(10):2734-2755. https://doi.org/10.1039/c9bm01809c

8. Wu G, Wu D, Lo J et al (2020) A bioartificial liver support system integrated with a DLM/GelMA-based bioengineered whole liver for prevention of hepatic encephalopathy via enhanced ammonia reduction. Biomater Sci 8(10):2814-2824. https://doi.org/10. 1039/c9bm01879d

9. Blanco-Fernandez B, Gaspar VM, Engel E et al (2021) Proteinaceous hydrogels for bioengineering advanced 3D tumor models. Adv Sci 8(4):2003129. https://doi.org/10.1002/advs.202003129

10. Yang S, Chen ZZ, Cheng YP et al (2021) Environmental toxicology wars: organ-on-a-chip for assessing the toxicity of environmental pollutants. Environ Pollut 268(Part B):115861. https://doi. org/10.1016/J.Envpol.2020.115861

11. Wang Y, Wu D, Wu G et al (2020) Metastasis-on-a-chip mimicking the progression of kidney cancer in the liver for predicting treatment efficacy. Theranostics 10(1):300-311. https://doi.org/ 10.7150/thno. 38736

12. Zhang B, Korolj A, Lai BFL et al (2018) Advances in organ-ona-chip engineering. Nat Rev Mater 3(8):257-278. https://doi.org/ 10.1038/s41578-018-0034-7

13. Skardal A, Devarasetty M, Soker S et al (2015) In situ patterned micro 3D liver constructs for parallel toxicology testing in a fluidic device. Biofabrication 7(3):031001. https://doi.org/10.1088/ 1758-5090/7/3/031001

14. Maharjan S, Cecen B, Zhang YS (2020) 3D Immunocompetent organ-on-a-chip models. Small Methods 4(9):2000235. https:// doi.org/10.1002/smtd.202000235

15. Lu SM, Zhang JW, Lin S et al (2021) Recent advances in the development of in vitro liver models for hepatotoxicity testing. Bio-des Manuf 4(4):717-734. https://doi.org/10.1007/ s42242-021-00142-7

16. Hassan S, Sebastian S, Maharjan S et al (2020) Liver-on-a-chip models of fatty liver disease. Hepatology 71(2):733-740. https:// doi.org/10.1002/hep.31106

17. Chen Z, Fu F, Yu Y et al (2019) Cardiomyocytes-actuated morpho butterfly wings. Adv Mater 31(8):1805431. https://doi.org/ 10.1002/adma.201805431

18. Wang H, Liu YX, Chen ZY et al (2020) Anisotropic structural color particles from colloidal phase separation. Sci Adv 6(2):eaay 1438. https://doi.org/10.1126/sciadv.aay 1438 
19. Vanaei S, Parizi MS, Vanaei S et al (2021) An overview on materials and techniques in 3D bioprinting toward biomedical application. Eng Regen 2:1-18. https://doi.org/10.1016/j.engreg.2020. 12.001

20. Eydelnant IA, Betty Li B, Wheeler AR (2014) Microgels ondemand. Nat Commun 5:3355. https://doi.org/10.1038/ncomm s4355

21. Ma C, Zhao L, Zhou EM et al (2016) On-chip construction of liver lobule-like microtissue and its application for adverse drug reaction assay. Anal Chem 88(3):1719-1727. https://doi.org/10. 1021/acs.analchem.5b03869

22. Lu S, Cuzzucoli F, Jiang J et al (2018) Development of a biomimetic liver tumor-on-a-chip model based on decellularized liver matrix for toxicity testing. Lab Chip 18(22):3379-3392. https:// doi.org/10.1039/c81c00852c

23. Pang L, Ding J, Liu XX et al (2019) Digital microfluidics for cell manipulation. Trac Trends Anal Chem 117:291-299. https://doi.org/10.1016/j.trac.2019.06.008

24. Samiei E, Tabrizian M, Hoorfar M (2016) A review of digital microfluidics as portable platforms for lab-on a-chip applications. Lab Chip 16(13):2376-2396. https://doi.org/10.1039/ c6lc00387g

25. Najah M, Griffiths AD, Ryckelynck M (2012) Teaching singlecell digital analysis using droplet-based microfluidics. Anal Chem 84(3):1202-1209. https://doi.org/10.1021/ac202645m

26. Au SH, Chamberlain MD, Mahesh S et al (2014) Hepatic organoids for microfluidic drug screening. Lab Chip 14(17):3290 3299. https://doi.org/10.1039/c4lc00531g

27. Theberge AB, Courtois F, Schaerli Y et al (2010) Microdroplets in microfluidics: an evolving platform for discoveries in chemistry and biology. Angew Chem Int Ed Engl 49(34):5846-5868. https:// doi.org/10.1002/anie.200906653

28. Liu Y, Sun L, Zhang H et al (2021) Microfluidics for drug development: from synthesis to evaluation. Chem Rev 121(13):74687529. https://doi.org/10.1021/acs.chemrev.0c01289

29. Kwapiszewska K, Michalczuk A, Rybka M et al (2014) A microfluidic-based platform for tumour spheroid culture, monitoring and drug screening. Lab Chip 14(12):2096-2104. https://doi.org/ 10.1039/c4lc00291a

30. Sakai Y, Hattori K, Yanagawa F et al (2014) Detachably assembled microfluidic device for perfusion culture and post-culture analysis of a spheroid array. Biotechnol J 9(7):971-979. https:// doi.org/10.1002/biot.201300559

31. Patra B, Chen YH, Peng CC et al (2013) A microfluidic device for uniform-sized cell spheroids formation, culture, harvesting and flow cytometry analysis. Biomicrofluidics 7(5):054114. https:// doi.org/10.1063/1.4824480

32. Karp JM, Yeh J, Eng G et al (2007) Controlling size, shape and homogeneity of embryoid bodies using poly(ethylene glycol) microwells. Lab Chip 7(6):786-794. https://doi.org/10.1039/ b705085m

33. Chen Q, Utech S, Chen D et al (2016) Controlled assembly of heterotypic cells in a core-shell scaffold: organ in a droplet. Lab Chip 16(8):1346-1349. https://doi.org/10.1039/c6lc00231e

34. Sakthivel K, O'Brien A, Kim K et al (2019) Microfluidic analysis of heterotypic cellular interactions: a review of techniques and applications. Trac Trends Anal Chem 117:166-185. https://doi. org/10.1016/j.trac.2019.03.026

35. Zhang W, Sun HC, Wang WQ et al (2012) Sorafenib downregulates expression of HTATIP2 to promote invasiveness and metastasis of orthotopic hepatocellular carcinoma tumors in mice. Gastroenterology 143(6):1641-1649. https://doi.org/10.1053/j. gastro.2012.08.032

36. Matsunaga T, Hosokawa M, Arakaki A et al (2008) High-efficiency single-cell entrapment and fluorescence in situ hybridization analysis using a poly(dimethylsiloxane) microfluidic device integrated with a black poly(ethylene terephthalate) micromesh. Anal Chem 80(13):5139-5145. https://doi.org/10.1021/ac800352j

37. Wu D, Yu YR, Zhao C et al (2019) NK-cell-encapsulated porous microspheres via microfluidic electrospray for tumor immunotherapy. ACS Appl Mater Interfaces 11(37):33716-33724. https:// doi.org/10.1021/acsami.9b12816

38. Oyaas J, Storro I, Svendsen H et al (1995) The effective diffusioncoefficient and the distribution constant for small molecules in calcium-alginate gel beads. Biotechnol Bioeng 47(4):492-500. https://doi.org/10.1002/bit.260470411

39. Tanaka H, Matsumura M, Veliky IA (1984) Diffusion characteristics of substrates in Ca-alginate gel beads. Biotechnol Bioeng 26(1):53-58. https://doi.org/10.1002/bit.260260111

40. Sasaki K, Akagi T, Asaoka T et al (2017) Construction of threedimensional vascularized functional human liver tissue using a layer-by-layer cell coating technique. Biomaterials 133:263274. https://doi.org/10.1016/j.biomaterials.2017.02.034

41. Qiu GY, Thakur A, Xu C et al (2019) Detection of gliomaderived exosomes with the biotinylated antibody-functionalized titanium nitride plasmonic biosensor. Adv Funct Mater 29(9):1806761. https://doi.org/10.1002/Adfm.201806761

42. Smith ZJ, Lee C, Rojalin T et al (2015) Single exosome study reveals subpopulations distributed among cell lines with variability related to membrane content. J Extracell Vesicles 4(1):28533. https://doi.org/10.3402/Jev.V4.28533

43. Liu Z, Shum HC (2013) Fabrication of uniform multi-compartment particles using microfludic electrospray technology for cell co-culture studies. Biomicrofluidics 7(4):44117. https://doi. org/10.1063/1.4817769

44. Zhu L, Kalimuthu S, Oh JM et al (2019) Enhancement of antitumor potency of extracellular vesicles derived from natural killer cells by IL-15 priming. Biomaterials 190-191:38-50. https:// doi.org/10.1016/j.biomaterials.2018.10.034

45. Lasli S, Kim HJ, Lee K et al (2019) A human liver-on-a-chip platform for modeling nonalcoholic fatty liver disease. Adv Biosyst 3(8):1900104. https://doi.org/10.1002/adbi.201900104

46. Selimovic S, Piraino F, Bae H et al (2011) Microfabricated polyester conical microwells for cell culture applications. Lab Chip 11(14):2325-2332. https://doi.org/10.1039/c1lc20213h

47. Ehrlich A, Tsytkin-Kirschenzweig S, Ioannidis K et al (2018) Microphysiological flux balance platform unravels the dynamics of drug induced steatosis. Lab Chip 18(17):2510-2522. https:// doi.org/10.1039/c81c00357b

48. Srigunapalan S, Eydelnant IA, Simmons CA et al (2012) A digital microfluidic platform for primary cell culture and analysis. Lab Chip 12(2):369-375. https://doi.org/10.1039/c1lc20844f

49. Pries AR, Secomb TW (2014) Making microvascular networks work: angiogenesis, remodeling, and pruning. Physiology 29(6):446-455. https://doi.org/10.1152/physiol.00012.2014

50. Jeong GS, da No Y, Lee J et al (2016) Viscoelastic lithography for fabricating self-organizing soft micro-honeycomb structures with ultra-high aspect ratios. Nat Commun 7:11269. https://doi. org/10.1038/ncomms11269

51. Zhang J, Zhao X, Liang L et al (2018) A decade of progress in liver regenerative medicine. Biomaterials 157:161-176. https:// doi.org/10.1016/j.biomaterials.2017.11.027

52. Maharjan S, Bonilla D, Sindurakar P et al (2021) 3D human nonalcoholic hepatic steatosis and fibrosis models. Bio-des Manuf 4(2):157-170. https://doi.org/10.1007/ s42242-020-00121-4

53. Zhou J, Liu W, Zhao X et al (2021) Natural melanin/alginate hydrogels achieve cardiac repair through ROS scavenging and macrophage polarization. Adv Sci 8(20):2100505. https://doi. org/10.1002/advs.202100505

54. Fang GC, Lu HX, De La Fuente LR et al (2021) Mammary tumor organoid culture in non-adhesive alginate for 
luminal mechanics and high-throughput drug screening. Adv Sci 8(21):2102418. https://doi.org/10.1002/advs.202102418

55. Chen CW, Kuo SM, Tien YC et al (2020) Steady augmentation of anti-osteoarthritic actions of rapamycin by liposomeencapsulation in collaboration with low-intensity pulsed ultrasound. Int J Nanomed 15:3771-3790. https://doi.org/10.2147/ IJN.S252223

56. Yang IH, Chen YS, Li JJ et al (2021) The development of laminin-alginate microspheres encapsulated with Ginsenoside $\mathrm{Rg} 1$ and ADSCs for breast reconstruction after lumpectomy. Bioact Mater 6(6):1699-1710. https://doi.org/10.1016/j.bioac tmat.2020.11.029
57. Zhu L, Kalimuthu S, Gangadaran P et al (2017) Exosomes derived from natural killer cells exert therapeutic effect in melanoma. Theranostics 7(10):2732-2745. https://doi.org/10.7150/thno. 18752

58. Lugini L, Cecchetti S, Huber V et al (2012) Immune surveillance properties of human NK cell-derived exosomes. J Immunol 189(6):2833-2842. https://doi.org/10.4049/jimmunol.1101988

59. Shoae-Hassani A, Hamidieh AA, Behfar M et al (2017) NK cellderived exosomes from NK cells previously exposed to neuroblastoma cells augment the antitumor activity of cytokine-activated NK cells. J Immunother 40(7):265-276. https://doi.org/10.1097/ CJI.0000000000000179

\section{Authors and Affiliations}

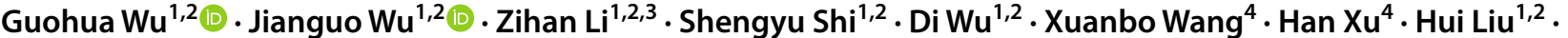 Yixiao Huang ${ }^{5,6} \cdot$ Rending Wang ${ }^{1,7} \cdot$ Jia Shen $^{1,7}$ (1) $\cdot$ Zhihong Dong $^{8} \cdot$ Shuqi Wang ${ }^{2,5,6,9}$}

1 The First Affiliated Hospital, School of Medicine, Zhejiang University, Hangzhou 310029, China

2 Institute for Translational Medicine, Zhejiang University, Hangzhou 310029, China

3 Department of Chemical Engineering, University of Waterloo, 200 University Avenue West, Waterloo, ON N2L 3G1, Canada

4 Department of Building Environment and Energy Engineering, Xi' an Jiaotong University, Xi' an 710049, China

5 National Engineering Research Center for Biomaterials, Sichuan University, Chengdu 610065, China
6 Clinical Research Center for Respiratory Disease, West China Hospital, Sichuan University, Chengdu 610065, China

7 Kidney Disease Center, The First Affiliated Hospital, School of Medicine, Zhejiang University, Hangzhou 310029, China

8 School of Mechanical Engineering, Chengdu University, Chengdu 610106, China

9 Institute for Advanced Study, Chengdu University, Chengdu 610106, China 